\title{
A RESULT ON SEMI-ARTINIAN RINGS
}

\author{
HAI QUANG DINH ${ }^{1}$ AND PATRICK F. SMITH ${ }^{2}$ \\ ${ }^{1}$ Department of Mathematics, Ohio University, Athens, OH 45701, USA \\ (haidinh@email.msn.com) \\ ${ }^{2}$ Department of Mathematics, University of Glasgow, \\ Glasgow G12 8QW, UK (pfs@maths.gla.ac.uk)
}

(Received 20 November 2001)

\begin{abstract}
It was shown by Huynh and Rizvi that a ring $R$ is semisimple artinian if and only if every continuous right $R$-module is injective. However, a characterization of rings, over which every finitely generated continuous right module is injective, has been left open. In this note we give a partial solution for this question. Namely, we show that for a right semi-artinian ring $R$, every finitely generated continuous right $R$-module is injective if and only if all simple right $R$-modules are injective.
\end{abstract}

Keywords: $V$ ring; semi-artinian ring; CS module; injective module

AMS 2000 Mathematics subject classification: Primary 16D50

Secondary 16P20; 16P60

We consider associative rings with identity, and all modules are unitary modules. A module $M$ is called a $C S$ module (or an extending module) if every submodule of $M$ is essential in a direct summand of $M$. Furthermore, a module $M$ is called a quasicontinuous module if $M$ is a CS module such that for any two direct summands $U, V$ of $M$ with $U \cap V=0, U \oplus V$ is also a direct summand of $M$. If $M$ is a CS module such that every submodule isomorphic to a direct summand of $M$ is itself a direct summand of $M$, then $M$ is called a continuous module. It is known that continuous modules are quasi-continuous.

A ring $R$ is called a right $V$ ring if every simple right $R$-module is injective. Further, a $\operatorname{ring} R$ is said to be right semi-artinian if every non-zero right $R$-module has a non-zero socle. Semi-artinian rings and modules were investigated, for example, in $[\mathbf{3}]$ and [5].

It was shown in [10] that if every continuous right $R$-module is injective, then $R$ is semisimple artinian. Motivated by this, rings $R$ with the following properties were discussed in $[\mathbf{9}]$.

$(p)$ Every finitely generated continuous right $R$-module is injective.

$(q)$ Every finitely generated CS right $R$-module is (quasi-) continuous.

The structure of rings satisfying either $(p)$ or $(q)$ is unknown. However, such a ring need not be semisimple artinian. It is shown in [9] that any simple right and left SI ring with 
zero socle is a ring with property $(p)$ (but not with $(q)$ ). Moreover, [7, Example 3.2] shows that rings satisfying $(p)$ and $(q)$ need not be semisimple artinian. Note that a ring $R$ is right $S I$ if every singular right $R$-module is injective (cf. [7, Chapter 3]). In this note we show that all right semi-artinian right $V$ rings satisfy both $(p)$ and $(q)$. For examples of such rings see [5].

Theorem 1. For a right semi-artinian ring $R$ the following conditions are equivalent:

(a) $R$ is a right $V$ ring;

(b) every finitely generated $C S$ right $R$-module is injective;

(c) every 2-generated CS right $R$-module is quasi-continuous; and

(d) every finitely generated continuous right $R$-module is injective.

To prove Theorem 1 we give a sufficient condition for a finitely generated CS module to have finite uniform dimension, in a general setting. A module $A$ is defined to be a $Q F D$ module if every factor module of $A$ has finite uniform dimension (cf. [1, p. 294] and [2]). Note that any noetherian or artinian module is QFD, and, more generally, so too is any module with Krull dimension by $[6,6.1(1)$ and 6.2(2)]. It is easy to check that if $Y$ is a submodule of a module $X$ such that $Y$ and $X / Y$ are both QFD, then $X$ is also QFD. Consequently, any finite sum of QFD submodules of a module is itself QFD. Note further that the sum $S_{1}(M)$ of all QFD submodules of a given module $M$ is a fully invariant submodule of $M$. Therefore, if $M=M_{1} \oplus M_{2}$, then $S_{1}(M)=S_{1}\left(M_{1}\right) \oplus S_{1}\left(M_{2}\right)$.

Proposition 2. Let $M$ be a finitely generated CS module such that for every proper submodule $K \subset M$, the factor module $M / K$ contains a non-zero $Q F D$ submodule. Then $M$ is a direct sum of finitely many uniform submodules.

Proof. As above, let $S_{1}(M)$ be the sum of all QFD submodules of $M$. By assumption, $S_{1}(M)$ is non-zero. Inductively we can define a $Q F D$ series $\left\{S_{\alpha}(M)\right\}$ of $M$ as follows: $S_{\alpha}(M)$ is a submodule of $M$ containing $S_{\alpha-1}(M)$ such that $S_{\alpha}(M) / S_{\alpha-1}(M)=$ $S_{1}\left(M / S_{\alpha-1}(M)\right)$. If $\alpha$ is a limit ordinal, then $S_{\alpha}(M)=\cup_{\beta<\alpha} S_{\beta}(M)$. By the assumption on $M$, there exists an ordinal $\gamma$ such that $S_{\gamma}(M)=M$. We call the least ordinal $\gamma$ with this property the QFD length of $M$.

We prove Proposition 2 by induction on $\gamma$. The statement is true for $\gamma=1$ because a finitely generated module $M$ with $S_{1}(M)=M$ is QFD, and hence of finite uniform dimension. Suppose that $\gamma>1$ and the result holds for all ordinals less than $\gamma$.

Assume on the contrary that $M$ does not have finite uniform dimension. Since $M$ is finitely generated, $\gamma$ cannot be a limit ordinal. Hence $\gamma-1$ exists and $M / S_{\gamma-1}(M)$ is the sum of its QFD submodules. Moreover, as $M / S_{\gamma-1}(M)$ is finitely generated, $M / S_{\gamma-1}(M)$ is a sum of finitely many QFD submodules. Therefore, $M / S_{\gamma-1}(M)$ has finite uniform dimension. Let $k$ denote the uniform dimension of $M / S_{\gamma-1}(M)$, and let $n$ be any integer greater than $k$. Since $M$ is CS (with infinite uniform dimension), we can decompose it as $M=M_{1} \oplus \cdots \oplus M_{n}$, where each $M_{i}$ does not have finite uniform dimension. Now, our above remark shows that $S_{\gamma-1}(M)=S_{\gamma-1}\left(M_{1}\right) \oplus \cdots \oplus S_{\gamma-1}\left(M_{n}\right)$. 
Hence $M / S_{\gamma-1}(M) \cong M_{1} / S_{\gamma-1}\left(M_{1}\right) \oplus \cdots \oplus M_{n} / S_{\gamma-1}\left(M_{n}\right)$. As the uniform dimension of $M / S_{\gamma-1}(M)$ is $k$, there must be an $M_{i}$ in the decomposition of $M$ that is contained in $S_{\gamma-1}\left(M_{i}\right)$. On the other hand, as a direct summand of $M, M_{i}$ is finitely generated and CS. Moreover, $M_{i}$ satisfies the other assumption about $M$. By the induction hypothesis, $M_{i}$ has finite uniform dimension. But this is a contradiction. Thus $M$ must have finite uniform dimension. As $M$ is CS, $M$ is a direct sum of finitely many uniform modules.

Corollary 3. Let $R$ be a ring such that for every proper right ideal $A$ there exists a right ideal $B$ containing $A$ such that $B / A$ is a non-zero $Q F D R$-module. Then every finitely generated $C S$ right $R$-module has finite uniform dimension.

Proof. Let $M$ be a finitely generated CS module. By hypothesis, every non-zero homomorphic image of $M$ contains a non-zero QFD submodule. Apply Proposition 2.

Corollary 4 (see Theorem 4.2 in [4]). A finitely generated CS right module over a right semi-artinian ring $R$ has finite uniform dimension.

Proof. By Corollary 3.

Proof of Theorem 1.

(a) $\Rightarrow$ (b). By Corollary 4, any finitely generated CS right $R$-module $X$ has finite uniform dimension. Hence by (a), $X=\operatorname{Soc}(X)$. This proves that $X$ is injective.

(b) $\Rightarrow(\mathrm{c})$. Clear.

(c) $\Rightarrow$ (a). Let $S$ be a simple right $R$-module with injective hull $S^{*}$. Suppose that $S^{*} \neq S$. Then there is a submodule $X \subseteq S^{*}$ containing $S$ such that $X / S$ is simple because $R$ is a right semi-artinian ring. Hence $X$ is cyclic and the composition length of $X$ is 2. Let $Y=S \oplus X$. By [6, 8.14], $Y$ is a CS module. But $Y$ is 2-generated, hence quasi-continuous by (c). Therefore, $S$ is $X$-injective (cf. [11, Proposition 2.10]). Thus $S$ splits in $X$, a contradiction. Hence we must have $S=S^{*}$, proving that $R$ is a right $V$ ring.

$$
\text { (b) } \Rightarrow(\text { d) } \Rightarrow(\text { a). Clear. }
$$

Remark 5. Proposition 2 was motivated by [4, Theorem 4.2] and its proof. Moreover, in $\left[4\right.$, Theorem 4.5], it was shown that if $R$ is a right semi-artinian ring such that $R_{R}^{(\mathbb{N})}$ is CS, then $R_{R}^{(A)}$ is CS for any set $A$, and hence $R$ is right and left artinian, and the injective hull of $R_{R}$ is projective.

Remark 6. Let $R$ be a right semi-artinian right $V$ ring. Then $R$ is von Neumann regular (see, for example, [6, 3.13(3)]). In particular, $R$ is semiprime. Therefore, each minimal right (left) ideal of $R$ is generated by an idempotent, and for each idempotent $e \in R, e R$ is a minimal right ideal if and only if $R e$ is a minimal left ideal. This implies that $\operatorname{Soc}\left(R_{R}\right)=\operatorname{Soc}\left({ }_{R} R\right)$. Let $S_{\alpha}^{l}$ denote the $\alpha$ th left socle of $R$. As $R / S_{\alpha}^{l}$ is von Neumann regular and right semi-artinian, we can inductively prove that $S_{\alpha}^{l}$ equals the $\alpha$ th right socle of $R$ for each ordinal $\alpha$. Hence $R$ is the union of its left socle series, and so $R$ is left 
semi-artinian (cf. [6, 3.12]). However, in general, $R$ is not left $V$ (see [8, Example 6.19]). This shows that the conditions (b) and (c) in our theorem are not left-right symmetric.

\section{References}

1. F. W. ANDERson AND F. R. Fuller, Rings and categories of modules, 2nd edn, Graduate Texts in Mathematics, vol. 13 (Springer, 1992).

2. V. P. Camillo, Modules whose quotients have finite Goldie dimension, Pac. J. Math. 69 (1977), 337-338.

3. J. Clark and P. F. Smith, On semi-artinian modules and injective modules, Proc. Edinb. Math. Soc. 39 (1996), 263-270.

4. H. Q. Dinh And D. V. Huynh, Some results on self-injective rings and $\Sigma$-CS rings, Commun. Alg., in press.

5. N. V. Dung And P. F. Smith, On semi-artinian $V$-modules, J. Pure Appl. Algebra 22 (1992), 27-37.

6. N. V. Dung, D. V. Huynh, P. F. Smith and R. Wisbauer, Extending modules (Pitman, London, 1994).

7. K. R. GoOdearl, The singular torsion and splitting properties, Memoirs of the American Mathematical Society, vol. 124 (1972).

8. K. R. Goodearl, Von Neumann regular rings (Pitman, London, 1979).

9. D. V. HuYnh, Some topics in ring theory, Lectures given at Department of Mathematics, Ohio University (Winter 2001).

10. D. V. Huynh and S. T. Rizvi, An approach to Boyle's conjecture, Proc. Edinb. Math. Soc. 40 (1997), 267-273.

11. S. H. Mohamed And B. J. MüLler, Continuous and discrete modules, London Mathematical Society Lecture Note Series, vol. 147 (Cambridge University Press, 1990). 